\title{
PAIRED TYPE III BURSTS
}

\author{
C. CAROUBALOS, J. HEYVAERTS, M. PICK, and G. TROTTET \\ Meudon Observatory, France
}

\begin{abstract}
Radioheliographic observations of type III bursts occurring by pairs in a repetitive fashion were made with the high time resolution E-W Nançay Radio heliograph $(169 \mathrm{MHz})$. The data were available in digitized form with a rate of $25 \mathrm{E}-\mathrm{W}$ profiles per second. The study is based on two days of observations: for the first one (January 14, 1971) the radio source was at $16^{\prime} \mathrm{W}$ and for the second one (June 29, 1971) at only $4^{\prime} \mathrm{W}$.
\end{abstract}

As seen in Figure 1, the time separations between the two components of the pairs

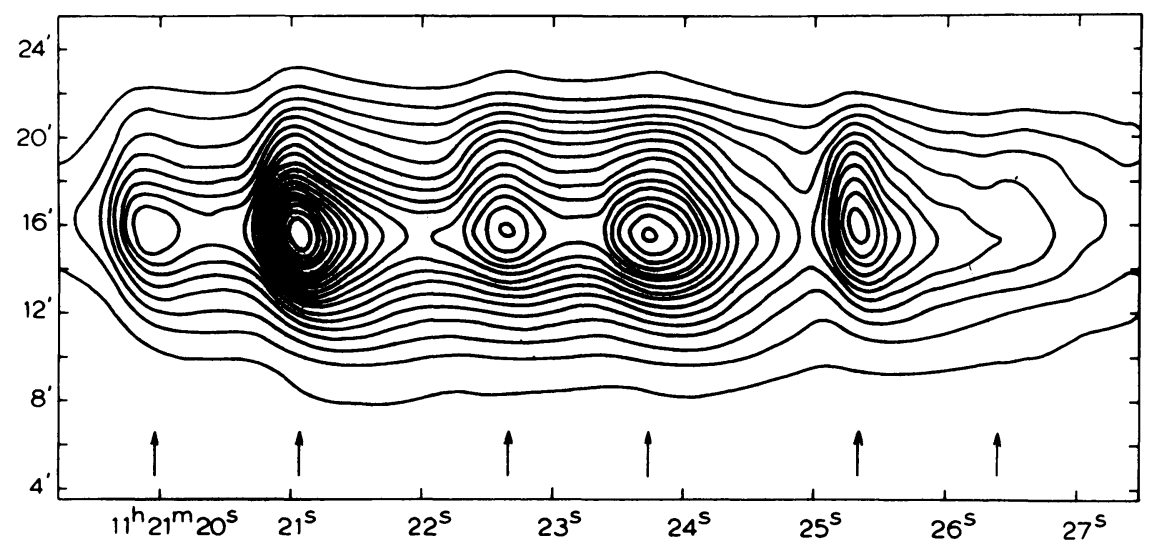

Fig. 1. January 14, 1971. E-W intensity distribution of the source of type III burst vs time observed at $169 \mathrm{MHz}$. The horizontal axis is universal time: the vertical axis indicates minutes of arc west of the central meridian. Arrows indicate maxima of elements of pairs.

are found to be maintained for the pairs of a given group. These time separations are found to be respectively $0.95 \pm 0.15 \mathrm{~s}$ and $0.3 \pm 0.05 \mathrm{~s}$ for the first and second days of observation whereas the corresponding time spacings between pairs are $2.4 \pm 0.4$ and $1 \pm 0.25 \mathrm{~s}$.

The two components of a paired burst do not systematically differ in any spacetime aspect: the durations, profiles, diameters and positions are found to be the same within the limits of the accuracy of our measurements. No specific law for the variation of the flux maxima is found, - the second component of the pair can be either weaker or stronger than the first one.

Different possible interpretations are reviewed: the hypothesis that the emission of both the fundamental and second harmonics is the cause of the pair cannot be accepted because of the similarity of the space-time properties of the source in both 
components of the pair seen at the same frequency. As no consistent relationship exists between the flux of the two components, it is also hard to accept an echo effect. Furthermore, a numerical simulation of such an effect for the second harmonic performed by the Monte-Carlo method proves that it is impossible to obtain the observed paired configuration even if only a slightly scattering corona is considered.

A possible explanation is suggested here: when a high current is being established in the lower atmosphere of the Sun, an oscillating regime is possibly set up, the period of which would be defined by the electric parameters of the circuit. The current flows in fine structures in which it would produce a pinching if the plasma is dense enough. The pinched plasma column will oscillate with a frequency determined by its own characteristics, perhaps, producing a beam of fast electrons at each maximum contraction. Calculations of the oscillations of such a plasma column for a given external sinusoidal current suggest that for reasonable conditions, especially certain periods of the external current oscillations, compressions of the column are obtained at intervals of several seconds. These may split into a pair more closely spaced at a typical time interval of about a second. Further development is necessary to test the validity of such an explanation.

\section{Reference}

Caroubalos, C., Heyvaerts, J., Pick, M., and Trottet, G. : 1974, Solar Phys. 37, 205.

\section{COMMENTS}

Smith: What accelerating mechanism have you in mind? You have only discussed the gross dynamics of the pinch. In fact, in all pinch experiments in the laboratory in which acceleration occurs, there are opposing fields and reconnection.

Pick: A pinched plasma column - the oscillations of which could produce beams of accelerated electrons at every maximum constriction. We did not try to present a detailed theory of the particle acceleration in the present study.

Stewart: Should not the decay rate hide everything? Maybe these events are not type III's.

Pick: At decimeter and meter wavelength, type III bursts are often very short. No confusion can exist between type III and pulsating structures. The latter are polarized and their source is not at the same position as the type III source.

Mullaly: Micro-wave bursts of duration smaller than $1 \mathrm{~s}$ appear to repeat themselves with a $10 \mathrm{~s}$ period. Maybe this could explain your meter wave observations?

Pick: Such periodicities of some seconds have effectively been observed in the microwave range. The time delay between two elements of a pair is in our case equal or less than $1 \mathrm{~s}$.

Cole: I have observed similar structures in type III bursts using the electro-optic spectrograph at Culgoora. 